\title{
Paratopie et entretien littéraire : Andreï Makine et Nancy Huston ou l'écrivain exilé dans le champ littéraire
}

Creative Nowhere: Paratopy and the literary interview. The case of Andrei Makine and Nancy Huston

\section{Valeria Pery-Borissov}

\section{OpenEdition Journals}

Édition électronique

URL : http://journals.openedition.org/aad/1629

DOI : 10.4000/aad.1629

ISSN : 1565-8961

\section{Éditeur}

Université de Tel-Aviv

\section{Référence électronique}

Valeria Pery-Borissov, «Paratopie et entretien littéraire : Andreï Makine et Nancy Huston ou l'écrivain exilé dans le champ littéraire », Argumentation et Analyse du Discours [En ligne], 12 | 2014, mis en ligne le 20 avril 2014, consulté le 21 décembre 2020. URL : http://journals.openedition.org/aad/1629 ; DOI https://doi.org/10.4000/aad.1629

Ce document a été généré automatiquement le 21 décembre 2020.

\section{cc)}

Argumentation \& analyse du discours est mis à disposition selon les termes de la licence Creative Commons Attribution - Pas d'Utilisation Commerciale - Pas de Modification 4.0 International. 


\title{
Paratopie et entretien littéraire : Andreï Makine et Nancy Huston ou l'écrivain exilé dans le champ littéraire
}

\author{
Creative Nowhere: Paratopy and the literary interview. The case of Andrei \\ Makine and Nancy Huston
}

Valeria Pery-Borissov

\section{Introduction}

Partie indéniable du «fait littéraire ", l'entretien constitue un pan de l'œuvre où l'auteur peut, en collaboration avec l'intervieweur, édifier une image de soi. Aussi divers travaux se sont-ils d'ores et déjà penchés sur les modalités discursives et interactionnelles au gré desquelles le genre de l'entretien autorise la construction d'une image d'auteur (Amossy 1997, 2007, 2010 ; Rodden 2007; Yanoshevsky 2004, 2006). Dans cette perspective, cet article se penche sur les entretiens d'auteurs exilés, pour interroger la spécificité de l'image d'auteur qui s'y met en place. Plus spécifiquement, il s'agira d'articuler l'entretien d'auteur et la notion de paratopie, c'est-à-dire le rapport simultané et paradoxal d'appartenance et de non-appartenance qu'entretient l'auteur avec la société d'accueil et le champ littéraire au sein duquel il écrit. Cette articulation est faite à partir des cas emblématiques de deux écrivains exilés en France, en l'occurrence Andreï Makine et Nancy Huston, l'un en provenance de Russie et l'autre du Canada, qui écrivent leurs œuvres en français et participent ainsi à la vie littéraire française. L'analyse de ces cas de figure nous permettra de montrer que, dans l'entretien, la paratopie de l'écrivain exilé se joue sur différents plans qui ne se contredisent pas mais qui se complètent pour aboutir à une paratopie dite créatrice de l'écrivain exilé. 
2 Les entretiens qui constituent le corpus analysé ont en général paru dans la presse française suite à la publication de nouveaux ouvrages des deux auteurs. Il s'agit des entretiens publiés entre les années 1995-2009, période où ils sont reconnus par les institutions littéraires françaises et où ils deviennent de plus en plus connus par le public des lecteurs. Parmi les écrits de Makine et Huston, on ne mentionnera que ceux qui permettent d'éclairer les phénomènes discursifs caractéristiques des entretiens, afin de voir s'il y a une spécificité de la figure de l'auteur exilé qui se dégage de ces textes à quatre mains.

\section{L'image de soi dans l'entretien et la paratopie}

3 L'entretien est le lieu où se construit l'image de l'auteur en interaction avec ce qu'on sait déjà de lui, et en interaction avec l'intervieweur (Yanoshevsky 2004). Rodden (2001) considère l'entretien comme " a form of literary performance ", c'est-à-dire comme un espace discursif où l'auteur exécute un ou plusieurs de ses rôles qui peuvent coïncider ou non avec l'image qu'on se fait de lui à partir de ses œuvres poétiques ainsi que de sa personne corporelle. Selon Yanoshevsky (2004), il s'agit dans l'entretien d'un affrontement, d'une sorte de jeu de forces entre l'image préexistante et l'image discursive ainsi qu'entre l'auteur et son intervieweur : "loin d'offrir à l'écrivain une simple occasion de se présenter sous un jour favorable, l'entretien fournit d'une part un cadre où s'affrontent la volonté de l'interviewé et les exigences de l'intervieweur et d'autre part l'image préalable de l'auteur et celle qui se construit lors de l'entretien. » (Yanoshevsky 2004: 6). Ce jeu de forces se produit suivant des conventions conversationnelles interactionnelles qui régissent le déroulement de l'entretien. Il importe, dans cet article, de s'interroger sur l'influence du statut personnel de l'écrivain, et notamment l'écrivain exilé, sur le déroulement de l'entretien : changeraitil les conventions d'interaction qui régissent ce genre? Susciterait-il un maniement spécifique de ces conventions? Y a-t-il des stratégies spécifiques activées par les participants dans le cas où l'interviewé est un auteur exilé qui écrit en français?

4 Même en écrivant dans la langue du pays d'accueil, les écrivains exilés restent étrangers à celui-ci. En même temps, éloignés physiquement de leur pays d'origine, ils ne lui appartiennent plus. On peut dire qu'ils se trouvent dans un non-lieu, ou un horslieu défini par un sentiment d'absence d'appartenance et par la nécessité de redéfinir les liens qui les lient à la société. Est-ce à partir de ce non-lieu que l'écrivain exilé construit son image de soi dans l'entretien? Pour répondre à cette question nous aurons recours au concept de " paratopie » défini comme une « localité paradoxale [...] qui n'est pas l'absence de tout lieu, mais une difficile négociation entre le lieu et le nonlieu, une localisation parasitaire, qui vit de l'impossibilité même de se stabiliser " (Maingueneau $2004: 52-53$ ).

5 Selon Maingueneau (2004: 85), les relations entre le créateur et la société et le créateur et l'espace littéraire peuvent être décrites comme une paratopie créatrice se jouant sur différents plans. Notamment, il distingue trois modalités selon lesquelles la paratopie créatrice peut s'exprimer :

Qu'elle prenne le visage de celui qui n'est pas à sa place là où il est, de celui qui va de place en place sans vouloir se fixer, de celui qui ne trouve pas de place, la paratopie écarte d'un groupe (paratopie d'identité), d'un lieu (paratopie spatiale) ou d'un moment (paratopie temporelle). 


\section{La paratopie d'identité: l'ethos de l'écrivain volontairement marginal}

\subsection{Se dire "passionnément exclu » de la société d'accueil : le cas d'Andreï Makine}

6 Andreï Makine, écrivain d'origine russe qui a fait sa carrière en France, s'impose dans le monde littéraire français en 1995 avec l'obtention du Prix Goncourt pour son roman Le Testament français ${ }^{1}$ publié chez Gallimard. C'est à partir de ce moment qu'on commence à l'intervieweur dans la presse quotidienne et hebdomadaire ainsi que dans les revues littéraires. La reconnaissance institutionnelle n'amène pas Makine à renoncer à son identification en tant qu'exilé, étranger. Il la souligne au contraire à travers les thématiques de ses œuvres fictionnelles, à travers ses interactions avec les journalistes, et jusque dans son accent russe prononcé. En même temps, il se distingue manifestement de la société russe actuelle en la critiquant et en refusant d'accepter les changements sociaux, culturels et mentaux qui y ont lieu. Dès lors que l'auteur insiste sur sa non-appartenance à la société d'accueil tout en clamant son refus d'appartenir à la société d'origine, dès lors qu'il choisit la langue étrangère comme langue d'écriture, son exil apparaît comme une paratopie par excellence: une paratopie spatiale, identitaire, linguistique.

7 Il n'est ainsi pas étonnant que même dans un entretien récent paru à la suite de la publication de son roman La Vie d'un homme inconnu (2009), Makine insiste explicitement sur le fait qu'il n'appartient pas à la société française :

Journaliste $^{2}$ : Vous sentez-vous définitivement intégré dans cette France où vous vous êtes fixé il y a plus de vingt ans?

AM : Intégré à la culture française, oui! Mais je ne vois pas pourquoi je devrais m'intégrer au politiquement correct qui règne dans le monde intellectuel d'aujourd'hui, à une société qui divinise les people et les footballeurs milliardaires au détriment de vrais penseurs et artistes, à un système économique où les financiers délinquants détruisent des millions d'emplois et donc des vies humaines. De tout cela, je me sens passionnément exclu. (Payot 2009 ; nous soulignons)

8 La question du journaliste n'est pas accidentelle: elle fait partie d'un échange où Makine prononce un plaidoyer en faveur de la Russie et s'exprime de façon critique sur la France. La réponse n'est pas non plus fortuite: bien que parfois de façon moins résolue, Makine se dissocie constamment de la société française contemporaine tout en s’identifiant à la culture française.

La question de l'intervieweur provoque Makine : par la façon dont elle est posée, cette question «fermée ${ }^{3}$ " incite l'interviewé à répondre tout d'abord par un "oui » ou «non », et elle oriente la réponse. En fait, Makine approuve d'abord son intégration par un « oui » exclamatif. Puis il s'explique et restreint la réponse initiale : s'il est prêt à s'identifier avec la culture française, il refuse décidément de s'adapter et s'adopter les mœurs imposées par la culture populaire contemporaine. A l'instar du «oui» exclamatif, la restriction est elle aussi émotionnelle grâce à l'emploi de l'adverbe "passionnément»; cet adverbe signale aussi le caractère intentionnel de la marginalisation de Makine, qui choisit de ne pas prendre part à certains aspects de la culture française. On voit dès lors comment l'entretien privilégie, dans le va-et-vient de 
l'échange, l'explicitation d'une idée déjà présente ailleurs chez l'écrivain. Si Makine se montre déjà critique - mais de façon détournée - dans ses essais et ses romans, ici, dans l'entretien, et grâce au questionnement de l'intervieweur, il expose l'aspect de l'exilé qui choisit parmi les valeurs de la société d'accueil celles qu'il estime respectables.

L'insistance sur son goût discriminatoire dans le choix d'intégration qui le classe parmi les «Français raffinés » apparaît aussi dans d'autres entretiens mais sur un ton qui pourrait être qualifié de provocant. Par exemple, lors de l'émission de Bernard Pivot, "Bouillon de culture ", où les invités sont interrogés sur leur vin français préféré, Makine déclare: "J'aime le champagne doux que les tsars russes buvaient [...] et les Français le détestent en général. » (Pivot 1995 ; nous soulignons). En plus de provoquer, Makine s'implique ici dans l'élaboration de son identité qui consiste en l'affiliation aux "tsars russes », c'est-à-dire en une identification au passé noble de la Russie que Makine oppose, à de nombreuses occasions, à la société russe contemporaine.

11 La prise de position subjective est exemplaire dans l'exemple suivant qui illustre l'attitude adoptée par Makine à l'égard de la société française et de ses représentants. Il s'agit cette fois-ci des aspects de la vie quotidienne :

J. : N'est-on pas déçu quand on a tellement rêvé de cette ville et qu'enfin, on y vit? $\mathrm{AM}$ : Non. Je vois Paris à travers mes yeux d'écrivain. Je refuse d'être entravé par le quotidien. C'est possible quand on n'a pas de famille, ce qui est mon choix. Depuis les prix, on me parle de cotiser pour la Sécurité sociale, pour la retraite. Je trouve cela burlesque. Les Français ne me déçoivent pas car je ne fais que les regarder. Je n'ai qu'un ami journaliste ici. Il a vécu en Algérie et est à moitié russe (Negrevergne 1995).

12 La question du journaliste focalise l'attention sur les émotions de l'auteur, mais le fait sous un ton provocant, en parlant de déception plutôt que de plaisir. Elle instaure une comparaison entre la ville actuelle et la ville rêvée de Makine, en faisant bien sûr référence à l'expérience personnelle de l'auteur. En répondant, Makine signale trois constituants paratopiques de sa condition d'exilé : être écrivain avant d'être Russe ou Français («à travers mes yeux d'écrivain»), se libérer des liens sociaux et matériels ( "Je refuse d'être entravé par le quotidien.»), ne pas se lier aux autochtones (« Il [son ami] a vécu en Algérie et est à moitié russe »). Il insiste ainsi sur son ethos critique extérieur, qui se pose comme supérieur, dû à la fois à son statut d'étranger et d'écrivain. Le sentiment de supériorité est exprimé par le biais de l'adjectif axiologique «burlesque » qui contient la disqualification de ce que la majorité des gens considèrent comme indispensable (famille, sécurité sociale, retraite). Le statut d'exilé de Makine s'étend alors au-delà des limites des différences culturelles entre deux pays et deux peuples, aux différences entre ceux qui acceptent une vie bourgeoise moyenne (la famille, les amis, le travail, la sécurité sociale) et ceux qui refusent de «jouer le jeu » et qui restent (comme le fait Makine) des marginaux.

\subsection{L'ethos d'une exilée déracinée: le cas de Huston}

13 Nancy Huston est une écrivaine francophone d'origine canadienne anglophone. Elle est auteure de plusieurs essais et romans, d'un livre de correspondances publié en collaboration avec Assia Djebar, de deux pièces de théâtre. Huston arrive à Paris en 1975 pour poursuivre ses études universitaires; elle rejoint les cercles gauchistes et féministes et commence à rédiger des essais ${ }^{4}$. Elle écrit alors en français. En 1980, Huston publie son premier roman, toujours en français ${ }^{5}$. En 1996, elle obtient le Prix 
Goncourt des Lycéens pour le roman Instruments des ténèbres et en 2006, le Prix Fémina pour Lignes de faille. Les entretiens avec Nancy Huston paraissent dans la presse française à partir l'année de sa consécration (1996) ; en 2000, elle prend part à un recueil d'entretiens consacré à des auteurs bilingues (Kroh 2000).

Dans ses entretiens, Huston élabore son statut d'exilée déracinée : elle reste étrangère dans la société d'accueil mais en même temps elle n'éprouve aucun sentiment de nostalgie ou d'attachement envers sa patrie. Elle le fait en commentant ses rapports, ou leur absence, avec les Français et avec le Canada.

L'échange suivant a lieu lors d'un entretien qui suit l'obtention par Huston du Goncourt des Lycéens, prix littéraire français «cadet» du Goncourt. Une série de brèves questions posées par l'intervieweur («Ecrivez-vous en français ou en anglais? Votre premier voyage en France? Paris aujourd'hui? Qui fréquentez-vous à Paris? Vous être française? Que pensez-vous des auteurs français actuels?») vise à proposer au lecteur une esquisse de l'écrivain étranger. Huston accepte de jouer le rôle de l'étrangère non intégrée car elle revient à plusieurs reprises sur le fait qu'elle a un meilleur contact avec les étrangers qu'avec les Français. C'est ainsi qu'elle insiste sur la distinction entre les autochtones et les exilés :

$\mathrm{J}:$ Qui fréquentez-vous à Paris ?

$\mathrm{NH}$ : Beaucoup d'étrangers. [...] Mon mari et moi sommes des exilés comme beaucoup dans notre cercle. Il y a très peu de Français. Ce n'est pas par sentiment anti-français mais cela nous donne une certaine liberté. Nous n'avons pas besoin de quêter cette approbation dont les Français sont très chiches (Poyet 1996).

Comme Makine, Huston souligne son altérité. Cependant, à la différence de Makine qui augmente toujours la distance entre lui et la société française en se positionnant à contre-courant de celle-ci, Huston semble se rapprocher de ses lecteurs, par le refus du ton péremptoire qui caractérise Makine: si, lors de l'échange cité ci-dessus, elle reproche aux Français d'être "chiches ", elle ne le fait pas dans le cadre d'une critique. Il s'agit plutôt d'expliquer pourquoi son cercle d'ami est constitué principalement d'étrangers, car « [...] les Français sont beaucoup moins tolérants [que les Américains] vis-à-vis de l'erreur ", comme elle l'avoue dans un autre entretien (Kroh $2000: 95)^{6}$.

Mais malgré la différence de ton avec Makine (l'un étant provocateur, l'autre modéré), elle présente quand même une affinité avec l'auteur d'origine russe, car tous les deux se montrent maîtres de leurs marginalité. Le fait de ne pas avoir de Français parmi ses amis ne témoigne pas d'une marginalité forcée comme c'est souvent le cas des immigrés mais contribue à la construction d'un ethos d'étrangère qui maitrise la situation, qui connaît bien le caractère des autochtones et qui fait ses choix en toute conscience.

18 Si pour Makine la paratopie joue au niveau du refus d'appartenance, celle de Huston est au contraire liée à l'absence : absence d'un sentiment de nostalgie lié au fait que sa vie au Canada était souvent associée au manque; manque d'une mère qui a quitté sa famille au profit d'une carrière professionnelle; manque d'événements et donc d'intensité de vie. Ainsi, la présence massive de la préposition "sans » qui apparaît chaque fois que l'auteur parle de son pays natal: "J'ai toujours vécu dans les régions sans guerre, sans bombes, sans drames, je n'ai connu ni la faim ni la répression politique » (Kroh $2000: 35$; nous soulignons).

Dans un contexte de la doxa occidentale, cette réponse de Huston semble valoriser l'absence des guerres et d'autres cataclysmes, et cela en raison du sens négatif que ces 
phénomènes ont dans la conscience collective de l'Occident. Cependant, replacée dans l'intertexte du discours de l'auteur exilé, cette absence d'événements acquiert une connotation « négative » :

$\mathrm{J}:[. .$.$] La déracinée que vous êtes éprouve-t-elle ce même type de nostalgie ?$

$\mathrm{NH}:$ Je n'éprouve pas la moindre nostalgie envers les paysages de mon enfance, pas plus que pour mon pays d'origine. Au contraire ! Lorsque, après avoir quitté l'ouest du Canada, en 1968, j'ai déménagé vers l'est des États-Unis, j'ai senti comme une intensification de la vie... une densification qui m'avait jusque-là manqué. Car l'Alberta incarnait, pour moi, la platitude de l'existence, ce vide qui m'angoisse. (Schiffer 2003)

La question du journaliste contient déjà une étiquette : "la déracinée que vous êtes » proposée d'ailleurs par Huston elle-même dans ses discours antérieurs. Elle ne provoque ainsi aucune réticence de la part de l'interviewée. En revanche, elle confirme et développe une idée de déracinement en expliquant ses raisons. On constate alors que pour ce qui concerne son pays natal, même les caractéristiques qui pourraient être perçues comme des avantages (pays calme et sûr) acquièrent chez Huston une valeur négative, et cela grâce aux mots appartenant au champ sémantique du manque comme « platitude » et « vide».

21 Le discours de l'entretien est très proche sur ce point de ses essais où l'auteur insiste aussi sur la platitude de son pays natal ${ }^{7}$ :

Comment faire comprendre à des Européens ce que signifie l'absence totale de ce qu'ils chérissent par-dessus tout : l'enracinement? [...] Même enfant, la réalité me semblait d'une fadeur et d'une homogénéité écœurantes: partout ce fut le règne des bons sentiments et du bon voisinage; partout était installée la platitude du neutre. $(1995: 178)$

Dans les deux discours, l'écrivaine exilée raconte son expérience personnelle et élabore ainsi un ethos d'exilée déracinée, qui ne s'identifie pas à son lieu de naissance ou d'enfance. Or, si l'essayiste rappelle son enfance dans le but unique de problématiser les différences entre les Européens et les Canadiens, dans l'entretien c'est l'interviewée en tant que personne et ses émotions qui occupent le centre. En conformité avec son ethos de porte-parole des immigrés qu'elle construit dans l'essai, Huston déclare son but qui est de «faire comprendre à des Européens ce que signifie l'absence totale de ce qu'ils chérissent par-dessus tout : l'enracinement ». Ainsi, elle oppose deux mentalités - des enracinés et des déracinés et tente d'en expliquer les différences. En tant qu'interviewée, par contre, elle ne généralise pas: elle élabore une image d'exilée contente de son exil qui la libère de son passé et des identités imposées par une appartenance définie et définitive. Elle s'écarte ainsi de l'opinion commune selon laquelle les exilés éprouvent une nostalgie envers la patrie quittée.

\subsection{L'ethos de « diseur de vérité» d'Andreï Makine}

L'ethos critique de Makine analysé ci-dessus constitue le fondement d'un ethos de "diseur de vérité » lié à la fois à son statut d'exilé et à la conception qu'a l'auteur du rôle de l'écrivain comme quelqu'un qui devrait «lutter contre les évidences rationnelles qui nous emprisonnent dans des définitions élaborées, imposées par la société » (Argand $2001: 25$ ).

Paveau (2010) souligne que la posture de "diseur de vérité » est risquée, étant donné qu'«il n'est pas si sûr que la vérité soit toujours considérée comme une chose bonne (à 
dire) et conforme à la morale». Ainsi celui qui l'adopte risque être relégué à la marginalité et même à l'exil (Paveau 2010a). Paveau note aussi qu'il existe plusieurs figures de diseurs de vérité, qui se distinguent par le champ dans lequel ils agissent et par l'époque de leur apparition ${ }^{8}$. Au-delà des distinctions qui différencient les cas de figures, le diseur de vérité est défini comme "porteur d'un discours marqué par le courage de la vérité, et [...] il court le risque d'être mis au ban de la société, car il publie des vérités insupportables » (Paveau 2010b).

La posture de "diseur de vérité » est inscrite d'ores et déjà dans la position marginale de l'exilé, capable de voir grâce à sa perspective externe les dysfonctionnements de la société d'accueil aussi bien que de sa patrie. C'est de cette position que Makine s'exprime sur les changements qui ont eu lieu en Russie juste avant et après l'écroulement de l'Union Soviétique, à l'occasion d'un entretien qu'il donne juste après l'obtention du Goncourt en 1995 :

AM : J'ai quitté l'Union soviétique parce que j'ai vu le pays tout entier basculer dans le capitalisme sauvage, et des intellectuels comme moi devenir des hommes d'affaires de la pire espèce. Cette perversion matérialiste m'a bouleversé (Nicolini 1995).

Il est évident qu'une telle vision est peu flatteuse pour la société russe ; pourtant, Makine tient à l'exprimer dans cette même interview, parce qu'il a «besoin de transmettre un message que j'estime important, ma vision du monde, une certaine conception de l'immortalité, de l'infini» (Nicolini 1995). Puisqu'une critique aussi explicite de la Russie n'existe pas dans ses autres écrits, il nous reste à présupposer que celle-ci est provoquée par le contexte « confessionnel » de l'entretien (où l'écrivain se trouve devant l'intervieweur-« prêtre », à qui il doit avouer "la vérité »). En tout cas, nous ne pouvons pas aller ici au-delà de l'hypothèse, étant donné que les questions sont omises dans la transcription.

Or les révélations concernant la situation actuelle en France ne sont pas spécifiques à l'entretien : elles apparaissent également dans l'essai. Où se situe donc la contribution de chaque genre à cette activité critique ? L'analyse de la réplique suivante en est très révélatrice :

$\mathrm{J}$ : Beaucoup d'anciens dissidents ou d'artistes de l'Est qui s'étaient élevés contre le communisme, d'Alexandre Zinoviev à Milan Kundera, se sont montrés également très critiques envers l'Occident une fois passés à l'Ouest. Quel regard portez-vous sur cela?

$\mathrm{AM}$ : Il y a des gens - je ne pense pas en particulier à Zinoviev ou Kundera - qui parfois sont affligés d'un caractère qui les pousse à critiquer tout régime. Tant mieux d'ailleurs. Mais est-ce tellement efficace? Ce que dit Zinoviev aujourd'hui sur l'Occident est un coup d'épée dans l'eau (Authier 2004).

La «consigne » introduite ici par le journaliste n'est pas univoque. Outre l'évocation d'un nouveau thème (critique de l'Occident par les exilés), elle renvoie implicitement l'interviewé à ses origines qui se trouvent, effectivement, en Europe de l'Est. Cependant, l'interviewé ignore cette allusion et élargit la discussion par le recours à la généralisation impersonnelle ( il y a des gens»). Par ce détour, il poursuit deux objectifs à la fois: d'abord, il rejette l'hypothèse du journaliste sur la causalité qui existe entre " être dissident de l'Est » et «critiquer l'Occident». Puis, il disqualifie les propos critiques tenus par ceux qui "sont affligés d'un caractère qui les pousse à critiquer tout régime ». Ainsi, l'interviewé se désolidarise et de l'intervieweur, et du groupe auquel ce dernier tente de le faire appartenir. Cette démarche paratopique de 
" diseur de vérité » solitaire par nature, s'accomplit dans la suite de la réplique citée où Makine propose sa propre vision du problème :

AM : Le problème aujourd'hui de la France, à mes yeux, est que ce pays veut de moins en moins être lui-même. Il y a en France le rejet de sa propre identité. On connaît cette identité. Il suffit de creuser un peu la terre pour percevoir l'héritage gréco-romain, gallo-romain, chrétien, judéo-chrétien, etc. Vous avez quand même un socle. Ou bien on arrache tout et on jette cela à la poubelle. Dans ce cas-là, on oublie tout, on peut porter le voile et commencer une autre civilisation. S'il n'y a pas de socle et si un pays ne veut plus être lui-même, comment peut-on intégrer les étrangers ? Corneille a tout dit : «Si vous n'êtes pas Romain, soyez digne de l'être ». Si l'on n'est pas Français, il faut être digne de l'être. C'est cela l'intégration [...] (Authier 2004 ; nous soulignons).

Dans cette analyse de la situation, l'interviewé remplit la fonction de « lanceur d'alerte [...] qui cherche à faire reconnaître, souvent contre l'avis dominant, l'importance d'un danger ou d'un risque " (Chateauraynaud 2008; nous soulignons). Il le fait à partir de son statut d'exilé, d'étranger qui fait partie de la société française et en même temps il ne s'intègre pas entièrement. Cette situation favorise donc les prises de position que les Français, trop absorbés par leur culture, ne peuvent pas et ne se permettent pas de faire, à cause de cet « ordre du discours » dont parle Foucault selon lequel tout discours est régi par un certain nombre de procédures dont l'interdit : « On sait bien qu'on n'a pas le droit de tout dire, qu'on ne peut pas parler de tout dans n'importe quelle circonstance, que n'importe qui, enfin, ne peut pas parler de n'importe quoi» (Foucault $1971: 364$ ). Un étranger comme Makine peut alors se permettre de dire ce que ne peuvent pas dire les Français de souche.

Si des idées semblables sont aussi présentes dans le discours essayistique de Makine, l'essayiste les exprime sur un mode moins assertif et plus politiquement correct. Dans l'entretien, l'interviewé adopte un ton plus agonique par le recours au vocabulaire provocateur comme «jeter à la poubelle ou arracher l'héritage». La provocation est aussi contenue dans un groupe verbal métonymique «porter le voile » qui signale de façon plus explicite que dans l'essai le groupe contre lequel est ciblée l'argumentation de Makine. Dans l'essai, le groupe problématique qui ne s'intègre pas et qui pose ainsi le problème à la société française est désigné par le terme plus vague de "jeunes $»^{9}$ : replacé dans le contexte social et culturel, ce mot peut être attribué à un groupe facilement identifiable, à savoir les habitants des banlieues parisiennes. Or, il ne renvoie pas aux attributs culturels de ces gens; en revanche, dans l'entretien Makine cible mieux sa critique en les désignant par le biais d'une marque de leur appartenance culturelle religieuse, le voile. Si nous supposons que c'est le genre d'entretien qui favorise la provocation, c'est pour deux raisons: d'une part, la présence de l'intervieweur qui formule les questions provocantes incite l'interviewé à donner des réponses révélatrices, d'autre part, le format même de ce genre où dans l'espace discursif et temporel très limité le locuteur qui voudrait transmettre un message, clarifier ses idées et construire une image de soi claire, radicaliserait son discours, choisirait les termes les plus frappants, et cela non seulement pour être entendu par le lecteur mais aussi pour insister sur un ethos indépendant à la fois des lieux communs d'une société donnée et de quelconques affiliations.

31 Cet ethos qui se situe dans le registre de la paratopie, c'est-à-dire hors de toute place fixe, est aussi repérable dans l'échange suivant où l'auteur exilé critique le champ littéraire français : 
$\mathrm{J}$ : Aujourd'hui, de nombreux écrivains étrangers installés en France en ont adopté la langue. Cela vous surprend-il?

$\mathrm{AM}$ : Je ne suis pas surpris, mais je reste très méfiant. Quand on pense au travail monumental sur soi que représente le passage d'une langue à une autre... Je reste persuadé qu'en France, de nombreux auteurs se font aider dans ce délicat travail et que certains textes sont tout simplement réécrits... Moi,j'ai au moins cette qualité : j'écris sans accent... (Clermont 2009 ; nous soulignons). paru en 2009, c'est-à-dire plus de dix ans après le Goncourt et la publication de plus d'une dizaine d'ouvrages en langue française. L'image d'auteur qui surgit à travers la question du journaliste est celle d'une autorité, d'un expert à qui on demande l'opinion. Makine accepte l'autorité conférée ${ }^{10}$. Dans sa réponse, toute en assumant tacitement son image préalable d'écrivain exilé, il la valorise par l'utilisation du qualificatif évaluatif « monumental » et il se détache simultanément des autres représentants de ce groupe («Moi, j'ai au moins cette qualité [...]»). Il gère ainsi sa paratopie d'écrivain exilé qui ne trouve cependant pas sa place dans le cercle d'écrivains issus, comme lui, des pays étrangers et écrivant en français.

Pourquoi l'auteur qui construit d'une manière pédante son argumentaire dans ses écrits, généralise-t-il dans les entretiens sur toutes sortes de thèmes ${ }^{11}$ sans parfois donner de preuves suffisantes à l'appui de ses assertions et sans tenter de s'en tenir aux normes de politesse convenues? Au niveau du fonctionnement du discours, c'est probablement le caractère dialogal du genre qui favorise chez Makine une certaine violence verbale (Kerbrat-Orrecchioni 1980) : la présence des intervieweurs déclenche la mise en mots de la provocation, de la transgression des règles de la bienséance. Ce qui en résulte, c'est que le comportement discursif de Makine contribue à la construction d'une image d'auteur exilé provocateur, "diseur de vérité » prêt à négliger le «bon ton" afin d'interpeller son interlocuteur. La dénonciation de la politique culturelle de la France ou/et des auteurs exilés qui écrivent "avec l'accent » permet ainsi à Makine de préserver sa paratopie dans la société dont il doit à la fois faire partie et se détacher.

\subsection{Se construire une image à travers la critique de la France}

L'analyse comparative des entretiens de Huston et de Makine montre une ressemblance d'opinion, par exemple sur le cercle intellectuel parisien ou sur l'état de la langue française. Or ils diffèrent, comme on l'a déjà vu, dans leurs styles respectifs, ce qui, à son tour, contribue à la construction d'ethè d'exilés différents.

Ainsi, à la différence de Makine qui se dissocie de façon consistante de la société d'accueil, Nancy Huston produit dans les entretiens l'image d'une exilée qui a réussi à s'intégrer dans son pays d'adoption, qui aime la France - son peuple, sa culture, sa langue. Cette image résulte de ce que l'écrivain raconte sur sa vie en France - sur sa participation active à la vie politique française (à titre d'exemple, elle a fait partie d'un groupe féministe français, elle a manifesté avec les gauchistes français, etc.), sur ses études en France (elle a participé aux séminaires de Barthes et de Lacan, sa thèse est consacrée à l'utilisation des gros mots en français ${ }^{12}$ ). Une lecture attentive des entretiens révèle néanmoins une identité plus complexe que ne laisse croire l'intégralité de ses écrits, où une image d'intégration réussie voisine avec un ethos critique et plus distant de la société d'accueil. Si leur style est différent, la critique de 
Huston à l'égard de la France et des Français reste toutefois proche de celle exprimée par Makine.

Par exemple, Huston exprime un nombre de réserves qu'elle a à l'égard des Français et de leur culture. Ces réticences apparaissent souvent à la suite des questions posées de façon explicite par les intervieweurs : «Paris aujourd'hui ? ", "Qui fréquentez-vous à Paris? ? ou encore «Vous êtes française? (Poyet 1996) comme dans l'exemple suivant :

$\mathrm{J}$ : Paris aujourd'hui?

$\mathrm{NH}$ : J'ai longtemps vécu rue des Rosiers. Le côté étranger, dans un pays étranger, me plaît. [...] Je l'ai quittée lorsque le hammam est devenu Chevignon. Je n'ai quand même pas quitté mes plaines pour retrouver les chapeaux de cow-boy ici! (Poyet 1996)

L'intervieweur adopte ici un style télégraphique qui rend possible une plus grande liberté d'interprétation de la question ${ }^{13}$ par l'interviewée parce qu'elle peut choisir l'aspect qui lui plaît et l'aborder dans sa réponse. Par exemple, cette question peut être complétée par «Aimez-vous le Paris d'aujourd'hui ? Comment trouvez-vous le Paris d'aujourd'hui en comparaison avec le Paris d'hier? Le Paris d'aujourd'hui, correspondil à la ville que vous vous imaginiez?"», etc. L'interviewée choisit d'exprimer une opinion critique concernant les changements culturels qui ont lieu à Paris, à savoir la perte du caractère historique et traditionnel du quartier remplacé par le représentant par excellence de la culture de consommation caractéristique de la société contemporaine/nord américaine. Cette critique de Huston fait écho à celle articulée par Makine qui reproche à la France d'avoir rejeté «sa propre identité14 » (Authier 2004). Pourtant, le changement qu'évoque ici l'interviewée ne concerne pas la perte de l'héritage culturel français au profit d'une culture de consommation identique dans tous les pays. À la différence de Makine, Huston ne plaide pas pour la conservation de la culture française (elle ne mentionne pas les indices de la francité) mais elle s'oppose au nivellement culturel, à l'effacement des différences culturelles. Cette prise de position est exprimée à travers l'usage de l'anecdote symbolique - le hammam remplacé par Chevignon, les plaines remplacés par les chapeaux de cow-boy. « Le hammam » et « les plaines » symbolisent ici la tradition et témoignent ainsi de la multiplicité culturelle, de la présence de différentes cultures dans la société française. Si Huston exprime une critique ici, il n'en reste néanmoins qu'elle ne part pas des mêmes présupposés que Makine. Alors que celui-ci se présente comme un descendant de la culture française d'antan rejetant de la sorte la culture française contemporaine, Huston, pour sa part, ne se montre pas favorable à une culture ou à une autre, et refuse ainsi la possibilité de rester enfermée dans une seule culture, une seule identité. Le résultat de ces différences est que Makine peut être associé aux traditionnalistes, voire aux nationalistes conservateurs, tandis que Huston se rapproche plutôt du multiculturalisme qui accepte la présence de différentes cultures au sein d'un même espace social.

Les jugements de deux auteurs se croisent aussi quand ils s'expriment de façon négative sur le cercle intellectuel parisien: Huston le traite de «monde clos et vaniteux " (Schiffer 2003) et Makine d'«hypocrites " et de "trafiquants d'idées" (Liedekerke $2000: 87$ ). Ainsi, ils portent un jugement négatif sur ce milieu ; la différence réside, à nouveau, dans une manière distincte de se situer par rapport au milieu critiqué. Si Makine dit habiter "à côté de Barbès » et ne jamais y voir « ces maitres à penser qui vivent dans les beaux quartiers " (Liedekerke $2000: 87$ ), Huston dit avoir appartenu jadis à ce cercle et assume ainsi sa propre critique : 
$\mathrm{NH}:[. .$.$] je suis critique à l'égard de mon gauchisme. [...] Comme d'autres gauchistes$ irresponsables, $\mathrm{j}$ 'ai contribué indirectement au maintien des régimes communistes dans le monde [...]. Je n'oublie jamais à quel point il est aisé d'avoir des idées, des opinions [...]. Il y a une chose incroyable en France : c'est la propension des gens à polémiquer bruyamment [...] (Argand 2001 : 34). Lycéens et où on commence à la reconnaître en tant qu'écrivain français. Huston ne possède pas encore l'autorité d'un auteur reconnu, sa légitimité dans le champ littéraire française est en train de se construire. Malgré ce statut encore incertain, l'interviewée n'essaye pas d'éviter la réponse à la question gênante du journaliste qui l'oblige à prendre position par rapport à ceux qui sont supposés avoir plus d'autorité et de légitimité dans le champ littéraire français. Elle s'exprime malgré cela de façon décidée en accusant implicitement les auteurs français d'être réactionnaires soumis au passé et aux autorités. Elle se distingue de ceux qu'elle décrit. Cette dissociation est repérable non seulement grâce à l'utilisation du lexique négativement connoté (« trop paralysés ») mais aussi grâce aux autres entretiens (par exemple, Kroh $2000: 96-97)$ où Huston exprime l'idée selon laquelle les écrivains exilés jouissent d'une plus grande liberté d'expression que leurs homologues autochtones. Ainsi, elle dit appartenir au camp des écrivains exilés.

Il est intéressant de comparer la réponse de 1996 avec celle de 2007 à une question similaire. On observe en fait un changement de ton :

$\mathrm{J}$ : Quel regard portez-vous sur la littérature française contemporaine?

$\mathrm{NH}:[. .$.$] En gros, mon sentiment intime est que (1) les écrivains français actuels ne$ se fatiguent pas, surtout la jeune génération. (2) Ils se donnent de petites tâches et les remplissent bien, mais personne ne semble avoir le courage de se lancer dans une entreprise d'envergure, une vaste fresque qui demanderait un effort sur la durée, impliquant non pas de parler de la solitude mais de vivre dans la solitude (Kuffer 2007).

On peut constater effectivement que le ton dans les deux exemples diffère. Bien que critique, la réponse de 1996 ne contient aucune ironie ou ambigüité, l'écrivain s'exprime de façon franche. C'est cette franchise qui est censée atténuer la critique difficilement acceptable de la part d'une étrangère. En revanche, dix ans plus tard, la réponse de Huston à une question similaire change au niveau formel tout en transmettant la même idée de fond. Ici, l'auteur a recours à l'ironie verbale ancrée dans la complémentarité de deux phrases qui critiquent les «écrivains français contemporains ». La phrase (1) contient une litote contenue dans l'expression « ne pas 
se fatiguer ", la phrase (2) est ironique grâce à la précision " et les remplissent bien » qui se réfère aux " petites tâches ».

Cette critique de Huston rejoint les paroles de Makine qui, dans ses entretiens, signale la futilité des thèmes traités par les auteurs français contemporains. Makine se dissocie explicitement de ces auteurs à propos desquels il s'exprime aussi avec une certaine hauteur: "Je n'arrive pas à prendre au sérieux les personnages qui souffrent parce qu'ils ne parviennent pas à faire l'amour plus de dix fois dans le mois.» (Argand 2001 : 25). On constate ici la présence de la première personne de l'indicatif (je) qui s'exhibe en assumant ainsi la responsabilité des énoncés agressifs. Dans l'exemple suivant, l'interviewé pousse à l'extrême son discours " politiquement incorrect ${ }^{15} »$ :

$\mathrm{J}$ : Quel regard portez-vous sur la littérature aujourd'hui ?

AM : Je trouve que les librairies sont pleines de non-livres. Ce sont des objets qui ont la forme d'un livre, mais qui n'en sont bien souvent pas. La télévision nous a rendu un service extraordinaire. Je suis un grand défenseur de la betise de la télévision parce qu'elle évacue tout ce qu'il y a de crétin en l'homme. Les écrivains en ont été libérés. Mais les auteurs sont têtus comme les ânes, ils continuent à écrire des petits romans mélodramatiques sans intérêt. Pourtant ce n'est plus la peine d'écrire là-dessus puisque la télévision se charge de l'idiotie permanente. (Thibeault 2004)

Le lexique choisi par l'interviewé - "têtus comme les ânes", "petits romans mélodramatiques sans intérêt ", «la bêtise » et «l'idiotie permanente »- a de fortes connotations négatives, voire il confère à l'énonciation un ton insultant. Ce ton traduit la condescendance avec laquelle l'interviewé perçoit la situation dans le champ culturel français. Cet effet est encore renforcé par l'ironie verbale contenue dans la juxtaposition des mots service extraordinaire, défenseur d'une part, et idiotie permanente, bêtise, crétin de l'autre.

Ainsi, les deux auteurs se positionnent en tant qu'étrangers sûrs d'eux, de leur compétence et de leur aptitude à se livrer à une analyse critique. Cette démarche est paratopique dans le sens où les auteurs inversent les positions fortes et faibles, ou «maximales et minimales» (Maingueneau 2004:96) et jouent des deux à la fois - le «statut minimal » d'un exilé et le «statut maximal » des écrivains institutionnalisés distingués par des Prix littéraires. Or ce statut maximal-minimal a des implications différentes chez les deux écrivains: Makine confirme ici son ethos de "diseur de vérité » qui exclut l'option de toute forme d'objection; la réponse de Huston, au contraire, invite à la discussion, et la présente ainsi comme prête à coopérer, ouverte au dialogue et à la discussion.

\section{La paratopie spatiale : préserver la non- appartenance pour pouvoir écrire}

La paratopie n'est pas affaire seulement de construction identitaire mais aussi des lieux choisis par un locuteur pour construire un espace à travers lequel il lui serait possible d'entretenir les rapports avec la société. Selon Mainguneau (2004: 103), «l'auteur, quelle que soit la modalité de sa paratopie, est quelqu'un qui a perdu son lieu et doit par le déplacement de son œuvre en définir un nouveau, construire un territoire paradoxal à travers son errance même ». 
C'est dans cette perspective qu'on doit analyser les répliques récurrentes dans les interviews de Makine où il avoue son goût pour les vagabondages dans les montagnes. Lorsqu'il est interrogé sur ces vagabondages, l'écrivain construit à la fois son ethos discursif et aussi une posture non-discursive en adoptant un certain comportement relatif à ce trait : il accepte, quoique sans enthousiasme ${ }^{16}$, que les journalistes (au moins deux dont nous avons les témoignages) l'accompagnent dans ses promenades. De plus, il les autorise ${ }^{17}$ à décrire en détail ses habitudes de randonneur : il dort « ici ou là à la belle étoile » sur « un matelas d'épines de pin » au lieu de sac de couchage, il ne planifie pas ses circuits ( Il ne sait jamais où ses vagabondages le mèneront »), sa nourriture est très simple - « Du fromage, des mûres et un peu de pain, cela suffit bien » (Roignant 1996). Par le biais de ces détails, Makine se présente comme un non-conformiste libéré des obligations et poussé seulement par l'amour du voyage.

Au début de son reportage, le journalise précise que Makine voyage ainsi « depuis son arrivée en France il y a huit ans» (Roignant 1996). Cette mention lie la posture de vagabond ${ }^{18}$ à son exil : les vagabondages semblent être le prolongement naturel de l'exil et lui confèrent un caractère permanent.

50 En racontant son amour du « voyage pédestre », Makine le lie non seulement à l'exil mais aussi à l'écriture :

$\mathrm{AM}$ : Le voyage pédestre à travers champs, sentiers et bords de mer est presqu'une tradition littéraire russe [...]. Autrefois, il y avait les «stranniki », des nomades un peu religieux qui vagabondaient dans la nature et vivaient en harmonie avec le cosmos. Ils s'arrêtaient ici ou là, chez des gens hospitaliers avides d'entendre leurs récits, de se nourrir de leurs aventures terrestres et mystiques. Pour moi, c'est une belle métaphore du lien qui existe entre l'écrivain et ses lecteurs. Ces derniers, pris par leurs obligations sociales, ne peuvent s'imprégner du cosmos, de cette dimension mystique, tandis que l'écrivain est un être libre qui, par son livre, leur apporte cette nourriture métaphysique (Roignant 1996).

51 Les mots-clés de cette citation - "tradition littéraire russe », "nomades », "libre », d'une part, et «aventures terrestres et mystiques », «s'imprégner du cosmos, de cette dimension mystique», "nourriture métaphysique», de l'autre, permettent de reconstruire la posture que l'auteur désire adopter, celle d'un écrivain libéré - par son exil et ses vagabondages - des obligations sociales. La liberté et le détachement à l'égard de la vie quotidienne sont représentés comme étant des atouts : c'est de cette façon-là que Makine construit le lieu paratopique qui donne raison à sa création ${ }^{19}$.

La paratopie spatiale est aussi présente dans les entretiens de Nancy Huston qui avoue, dans un entretien donné à Vogue à la suite de la publication de son roman Dolce agonia (2002), son besoin de s'isoler pour pouvoir écrire :

$\mathrm{J}$ : Une journée de travail type?

$\mathrm{NH}$ : Je quitte la maison pour mon studio à 9 heures. Dans cet endroit, je n'ai pas de téléphone, pas d'Internet. Tout en faisant l'éloge du lien, je dis fortement que j'ai besoin de m'isoler pour travailler. Impossible pour moi d'écrire au milieu de la famille. Dans ce bureau, je n'ai pas de photos de mes enfants, je ne suis la femme de personne, l'amie de personne. Je ne suis même pas un écrivain ; c'est à la maison que je gère ma carrière, entre guillemets (Binet 2004).

53 La description de ce studio fait penser à une île qui, quant à elle, représente un espace isolé : « elle matérialise en effet l'écart constitutif de l'auteur par rapport à la société. [...] l'île appartient au monde sans y appartenir » (Maingueneau 2004 : 103). En fondant dans cette réponse au journaliste sa paratopie, Huston lui donne raison d'être (elle en a besoin pour pouvoir écrire) et fait en même temps la distinction entre l'écriture en tant 
que l'activité créatrice et l'inscription dans le champ littéraire (« c'est à la maison que je gère ma carrière ").

Ainsi, en ce qui concerne la paratopie spatiale, les deux auteurs en question la maintiennent dans leurs discours d'entretien respectifs mais ils ont recours à des embrayeurs paratopiques différents: Makine se sert de l'image d'un errant qui ne peut pas s'enfermer dans un lieu, Huston opte pour une chambre enfermée, sorte d'une île qui se trouve à la fois dans et hors du monde.

\section{La paratopie créatrice : l'ethos de poète solipsiste}

Selon Maingueneau, « est créateur celui qui a organisé une existence telle qu'il peut y advenir une œuvre, la sienne » (Maingueneau 2004 : 92). Dans le cas des écrivains exilés comme Makine et Huston, cette organisation consiste en une insistance sur une paratopie identitaire et spatiale qui aboutit à une paratopie créatrice, celle donc qui subordonne toute existence d'une personne à la création. Nous tenterons de le montrer dans ce qui suit.

Dans ses entretiens, Makine insiste sur son identité russe et se distancie de la société française. Il revendique simultanément une identité française, le français étant la langue de sa grand-mère. Aussi pense-t-il dans les deux langues, il fait un plaidoyer pour l'intégration et participe de façon active à la vie littéraire et culturelle française (les entretiens, les prix, la reconnaissance). Cette ambivalence du discours de Makine motive des questions d'éclaircissement sur son appartenance :

1) J : Vous avez désormais la nationalité française. Comment vivez-vous entre deux mondes?

AM : Je vis dans mon monde. Dans mon univers à moi (Vavasseur 1998).

2) J : On a parlé de vous comme d'un Russe s'exprimant en français. Cette définition vous convient-elle?

$\mathrm{AM}: \mathrm{Je}$ pense que dès que l'on commence à écrire on devient étranger... (Liedekerke $2000: 86)$.

3) J : Vous avez désormais la citoyenneté française. Avez-vous le sentiment d'avoir trahi quelque chose de vos racines?

AM : Non, car je n'ai vraiment qu'une seule patrie : l'écriture... (Barillé 2001).

57 Les questions posées par les journalistes sont plus ou moins identiques dans les trois exemples : en fonction d'un savoir préalable sur le statut de l'interviewé, ils demandent à Makine de le commenter. Les réponses de Makine se ressemblent aussi, or chaque fois elles deviennent de plus en plus précises de façon à ce qu'on comprenne que l'écrivain, selon le cliché romantique, n'est nulle part chez soi à part dans l'écriture. Il se présente ainsi comme " quelqu'un dont l'énonciation se constitue à travers l'impossibilité même de s'assigner une véritable place, qui nourrit sa création du caractère radicalement problématique de sa propre appartenance au champ littéraire et à la société" (Maingueneau 2004 : 85).

Il s'ensuit que la question d'appartenance nationale, ethnique ou langagière perd toute pertinence et cède la place à des critères poétiques, comme dans l'exemple suivant :

$\mathrm{J}$ : Pourquoi avoir choisi d'écrire en français?

AM : Je m'intéresse aux Français tout naturellement. Je voulais m'adresser à eux et dans quelle autre langue aurais-je pu le faire? De plus, si j'avais écrit en russe, j'aurais été traduit et donc trahi. Mais le choix de la langue n'est pas important. Ce 
qui est important c'est de garder ce langage poétique parce que la vision que l'on a, on peut l'exprimer dans n'importe quelle langue... (La Gazette 2004). plupart de ses prises de paroles, une identité multiple. La réponse de l'interviewée semble paradoxale : d'une part, elle confirme la suggestion de journaliste - « Multiple, oui » et ainsi elle dit avoir plusieurs identités à la fois; d'autre part, elle dit se sentir « ni... ni » et donc ne s'approprier aucune identité, elle n'aurait pas alors un chez soi ? Le paradoxe se résout quand on lie cette suggestion à sa fonction paratopique (Maingueneau 2004) d'écrivaine pour laquelle «c'est un immense atout [...] d'être de nulle part, parce que le roman naît lorsque l'on comprend que l'identité n'est pas une chose donnée » (Frey 2006). Autrement dit, «être de nulle part » c'est être dans le monde de l'écriture et construire, à partir de ce monde, des identités.

Les deux auteurs érigent ainsi l'absence d'appartenance en condition nécessaire de la création littéraire. Cette démarche permet alors une construction d'une image de l'écrivain qui n'est pas spécifique à l'exil. Ainsi, Makine l'essentialise au-delà des distinctions entre les exilés et les autochtones :

$\mathrm{J}:$ Ne seriez-vous pas un résistant?

AM : L'écrivain a le pouvoir de recréer le temps, de l'anéantir, de le dominer par les mots. Le pouvoir de recréer l'être aussi selon sa propre expérience. Il est le seul à pouvoir transfigurer la réalité, c'est-à-dire à la voir telle qu'elle est sous la couche plaquée or ou argent ou bronze que vous montrent d'un côté la télévision et de l'autre des intellectuels asservis au discours politique, médiatique, sociologique. La sous-culture inonde les ondes et les écrans. En promettant le bonheur, des chansons, des millions, elle fonctionne comme une drogue mentale, la même que celle dont parle le grand inquisiteur de Dostoïevski. La littérature est le dernier carré de résistance face aux machines à crétiniser. [...] Écrire, c'est un choix métaphysique, une transfiguration, un investissement total. On devient autre et si ce n'est pas vécu ainsi vous n'êtes qu'un bon faiseur de romans (Argand 2001 : 25-26).

Aucun mot de la réponse de l'interviewé ne se réfère à son appartenance ethnique. Parce qu'en fait si l'écrivain a un pouvoir de "transfigurer la réalité » et de "recréer l'être ", aucune étiquette n'aurait de sens. Le choix d'être écrivain prédomine alors sur le statut d'exilé : cette prédominance est inscrite par le biais d'un rythme ternaire de la phrase «Écrire, c'est un choix métaphysique, une transfiguration, un investissement

Argumentation et Analyse du Discours, 12 | 2014 
total. ». Ces trois qualifications de l'écriture la placent au-delà de toute autre activité humaine, et donc au-delà de la distinction entre l'exilé et l'autochtone. Ce qui compte alors pour Makine c'est la conscience qu'a l'écrivain de son rôle défini par ailleurs dans la question du journaliste, à savoir le rôle d'un résistant qui est seul à voir le sens caché «sous la couche plaquée or ou argent ou bronze que vous montrent d'un côté la télévision et de l'autre des intellectuels asservis au discours politique, médiatique, sociologique ». Les termes par lesquels Makine qualifie ici ce à quoi l'écrivain s'oppose, à savoir la " sous-culture ", " machines à crétiniser ", renvoient à l'ethos polémique de " diseur de vérité » analysé supra. Or ici cet ethos n'est pas lié au statut de l'exilé mais à celui de l'écrivain. Cette élimination de l'ethos d'exilé au profit de celui d'écrivain est spécifique aux entretiens de Makine. Elle est rendue ici possible grâce à l'interaction avec le journaliste dont les questions se détachent du questionnement lié au statut d'exilé au profit des éthè construits dans le discours de Makine.

On retrouve un processus semblable dans les entretiens de Huston qui décrit l'expérience d'écriture en termes très proches de ceux de Makine :

$\mathrm{J}$ : Est-ce que de cette précoce mais intense activité de votre imagination qu'est né ce rapport parfois difficile que vous entretenez avec la réalité ? [...]

$\mathrm{NH}$ : Oui. L'écriture est, pour moi, une sorte d'exploration, illimitée. C'est une manière de dépasser, voire transcender le monde réel, qui est souvent décevant et où j'étouffe parfois. L'écriture, pour moi, est donc, parce que ses possibilités sont infinies, un vrai acte de création [...]. On peut donc, par échafaudage des scénarios les plus fous, y corriger et même y améliorer le monde. C'est cela qui, dans l'écriture, est exaltant! La littérature n'est donc, pour moi, que cet immense espace de liberté, où je connais aussi bien (ce sont là mes multiples identités) l'enfer que le paradis, le Diable que Dieu... (Schiffer 2003)

La question ne se réfère aucunement à l'exil de Huston ; c'est ce qui permet sans doute à celle-ci d'en faire abstraction dans sa réponse. A l'instar de Makine, elle signale le pouvoir qu'a l'activité d'écriture de « dépasser, voire transcender le monde réel » et de « corriger et même $[y]$ améliorer le monde ». En disant que l'écriture est un « immense espace de liberté ", Huston rapproche cette partie de son identité de celle de l'exil car l'exil est aussi perçu par Huston comme un espace de liberté. Or le choix des verbes « dépasser » et «transcender » présente l'écriture comme supérieure à l'exil en termes de liberté. Si l'exil a libéré Huston d'une identité unifiée et lui a permis de s'ouvrir à la multiplicité identitaire, l'écriture lui permet de s'approprier cette multiplicité : elle y connait « aussi bien (ce sont là mes multiples identités) l'enfer que le paradis, le Diable que Dieu ». Huston valorise ainsi son ethos d'écrivain et légitime le refus d'appartenir « du tout au tout » à une société précise.

\section{Conclusion}

66 À travers l'analyse des entretiens de Makine et de Huston, nous avons souhaité examiner les modalités selon lesquelles se joue la construction de l'image de soi d'un écrivain exilé dans l'entretien d'auteur. C'est en effet par le biais de l'embrayage paratopique que les deux écrivains construisent une image de soi à multiples visages, tous unis sous le signe de la paratopie : l'ethos de "diseur de vérité » ou de "vagabond » de Makine, l'ethos d'une adepte de multiculturalisme ou d'une " exilée déracinée » de Huston, enfin, un ethos critique qui les placent dans une paratopie de différents ordres identitaire, spatiale, créatrice. C'est ce non-lieu qui leur permet de convertir leur 
intenable situation d'exilé en un lieu où la prise de parole et de position devient possible. On peut dire que la mise en place de la paratopie légitime le statut des auteurs exilés : le fait qu'ils soient étrangers n'est plus considéré comme un défaut ou un manque. Il s'agit plutôt d'un statut particulier, avantageux qui élargit leur champ de vision et leur potentiel créateur.

On peut se demander si cette démarche est particulière aux écrivains exilés. Or Maingueneau (2004) montre déjà que la paratopie est intrinsèquement liée à la création et l'énonciation d'écrivain. Ainsi, « cette intenable situation dans laquelle se trouve et se place l'écrivain pour devoir écrire et pouvoir ainsi supporter par son écriture ce que sa situation a d'intenable est tout aussi bien, dans une conjoncture très différente, celle» de Proust, de Céline, de La Bruyère, de La Fontaine, de plusieurs autres. Cependant, Maingueneau observe le fonctionnement du concept à travers l'analyse des œuvres littéraires, alors que nous avons tenté de le montrer par les entretiens littéraires. L'analyse de la manière dont la paratopie s'est élaborée dans l'entretien d'auteur exilé et la manière dont elle imprègne les images de soi des écrivains exilés montre qu'une priorité est donnée chez les écrivains interviewés que nous avons présentés ici au statut d'écrivain, au détriment de celui d'exilé. Il s'agit de montrer que l'entretien devient un lieu de légitimation du statut de l'auteur exilé qui n'essaye pas de dissimuler son altérité mais qui en fait, au contraire, la source de son identité littéraire.

C'est souvent grâce aux questions des intervieweurs concernant les rites d'écriture et l'attitude de l'écrivain envers la société et le champ littéraire d'accueil que les écrivains en question peuvent fonder cette paratopie qui leur octroie leur droit et leur raison d'existence dans la société et dans le monde littéraire français: en fait ils ne dissimulent pas leur altérité et en même temps ils mettent au premier plan leur essence d'écrivain. Dans les deux cas - quand il s'agit de leur parler de leur altérité, et quand on discute de leur écriture -, la paratopie est en jeu. Les questions des intervieweurs facilitent cette double élaboration de paratopie : d'une part, elles renvoient souvent les écrivains en question à leurs origines. D'autre part, elles permettent aux écrivains de se détacher dans leurs réponses respectives du discours de l'exil en accentuant le caractère paratopique de la situation de l'écrivain, de tout écrivain, indépendamment de l'exil. C'est donc dans cette dialectique qui s'instaure dans le dialogue entre intervieweur et interviewé, dialogue où des questions sur la pratique de l'écriture et sur son statut d'exilé, permettent à l'écrivain de fonder sa propre place originale, créatrice, toute en s'éloignant du créneau marginal qui aurait pu être le sien dans le champ littéraire.

\section{BIBLIOGRAPHIE}

Amossy, Ruth. 1997. " Autoportrait à deux voix : La nuit sera calme de Romain Gary », Elseneur, Numéro spécial Ecriture de soi et dialogue, sous la direction d'A. Goulet, pp 141-162

Amossy, Ruth. 2014 [2007]. « Le 'Divin Dali' du visuel au verbal : Autoportrait et interaction dans le livre-entretien » (dans le présent numéro) 
Huston, Nancy. 1995. « La Rassurante Étrangeté », Désirs et réalités. Textes choisis 1978-1994 (Ottawa : Leméac), 177-185

Huston, Nancy. 1996. Instruments des ténèbres (Arles : Actes Sud)

Huston, Nancy. 2001. Dolce agonia (Arles : Actes Sud)

Huston, Nancy. 2006. Lignes de faille (Arles : Actes Sud)

Maingueneau, Dominique. 2004. Le discours littéraire. Paratopie et scène d'énonciation (Paris : Colin)

Makine, Andreï. 1995. Le testament français (Paris : Mercure de France)

Makine, Andreï. 2009. La vie d'un homme inconnu (Paris : Seuil)

Negrevergne, Cécile. 1995. « Andreï Makine : 'Il faut se sacrifier pour ses lecteurs' », TELE 7 Jours (22 décembre)

Payot, Marianne. 2009. « Makine contre les 'russophobes' », Le Figaro (8 janvier)

Pivot, Bernard. 1995. « Bouillon de culture avec William Christie, ToshiTakata, Ruppert Everett, Alfredo Arias et Andreï Makine », TV5 (22 décembre)

Rodden, John. 2007. Performing the Literary Interview. How writers craft their public selves (Lincoln \& London : University of Nebraska Press)

Yanoshevsky, Galia. 2004. «L'entretien d'écrivain et la co-construction d'une image de soi : le cas de Nathalie Sarraute », Revue des sciences humaines, 273, 131-148

\section{NOTES}

1. C'est aussi son unique roman traduit en russe. Il a été publié dans une revue littéraire russe et n'a pas eu de grand succès. Ainsi, Makine est pratiquement absent du champ littéraire russe.

2. Les paroles des journalistes seront signalées par J ; celles des auteurs par AM pour Makine et $\mathrm{NH}$ pour Huston.

3. Les questions fermées autorisent seulement une réponse de type «oui, non»; elles contiennent un maximum de présupposés, car le contexte est contenu dans la question.

4. Parmi ses premiers essais: «A Monique, lettre qui voudrait ne pas rester morte» (1978), « Anatomies et destins » (1978), « La main morte » (1978), recueillis dans Huston 1995.

5. Ce n'est qu'en 1993 qu'elle commence à écrire aussi en anglais. Dès lors, elle se dit être une auteure bilingue qui alterne la langue d'écriture et se traduit elle-même.

6. Le discours critique est modéré ici par la comparaison : l'assertion de Huston n'est pas absolue mais relative aux éléments choisis par la locutrice. La modération rend le discours de Huston plus accessible au lecteur français qui se sent moins offensé qu'en lisant les remarques provocantes de Makine.

7. Il faut signaler que ce discours sur la fadeur de l'Alberta est aussi présent dans son roman Cantique des plainesà propos duquel l'interviewée avoue vouloir « faire coïncider » la forme et le fond:

$\mathrm{J}$ : Est-ce que le fait d'avoir retrouvé l'anglais a été l'un des éléments déclencheurs de ce style très lyrique qui est celui des Cantique des plaines?

$\mathrm{NH}:[. .$.$] Je voulais en quelque sorte faire coïncider la syntaxe et le paysage. Je voyais comme des$ champs de blé à perte de vue et je voulais que la phrase porte cette idée d'éternité... » (GirardDaudon et Bernard 2001 : 7).

De plus, elle signale qu'à travers le caractère de Paddon, personnage de Cantique des plaines, elle décrit la nature fade et plate de l'Alberta. 
8. Voir, pour plus de détails, les cinq premiers articles de Paveau publiés sur son blog en automne 2010, http://penseedudiscours.hypotheses.org/category/series-de-saison/ automne-2010-les-diseurs-de-verite

9. «[...] le vieil homme qui habite avec son épouse dans une maison particulière à Roubaix, est pris à partie par ce qu'on appelle en France "des jeunes". [...] quelques mois auparavant, en plein jour, dans un parc de Nice, deux "jeunes" tuent une mère sous les yeux de son fils de cinq ans. [...]. » (Makine 2006 : 94-95).

10. En effet, dans les entretiens antérieurs, c'est Makine lui-même qui s'approprie cette autorité en adoptant souvent un ton autoritaire. Le fait que dans l'exemple présent c'est l'intervieweur qui lui confère de l'autorité témoigne sans doute du changement du statut de l'écrivain aux yeux des représentants des institutions littéraires françaises qui l'incluent, de la sorte, parmi ceux qui jouissent d'un pouvoir dans le champ littéraire français.

11. À part les auteurs français contemporains, la littérature française et l'utilisation de la langue, on trouve, par exemple, le jugement suivant : «Pour Pouchkine et d'autres, l'art était un monde supérieur. On ne parlait jamais de l'argent. Il y avait une mission, une vocation, un sacerdoce. En France, on ne parle que d'argent. » (Authier 2004); ou encore, à propos du milieu journalistique : « À deux reprises, j'ai proposé des articles à la presse française. Mais ils ont été refusés. C'est un milieu où il ne faut pas essayer de percer quand on n'a pas un plan de carrière bien défini. » (Le Fol 1998).

12. La thèse de doctorat d'Andreï Makine est consacrée à l'œuvre d'Ivan Bounine, écrivain russe émigré en France. Ce détail extra-discursif (car Makine ne le mentionne jamais dans ses écrits et dans ses entretiens) reflète les différences qui existent entre les deux auteurs : alors que le choix du thème de doctorat de Huston reflète le désir de faire partie de la société française, le choix de Makine souligne, en revanche, son attachement à la culture russe.

13. Les raisons qui conditionnent l'adoption de ce style peuvent être purement techniques, à savoir les limites temporelles et/ou spatiales. Cela n'a pas d'importance dans le sens où une question ainsi formulée confère un degré plus grand de liberté à l'interviewé.

14. AM : Le problème aujourd'hui de la France, à mes yeux, est que ce pays veut de moins en moins être lui-même. Il y a en France le rejet de sa propre identité. On connaît cette identité. Il suffit de creuser un peu la terre pour percevoir l'héritage gréco-romain, gallo-romain, chrétien, judéo-chrétien, etc. Vous avez quand même un socle. Ou bien on arrache tout et on jette cela à la poubelle. Dans ce cas-là, on oublie tout, on peut porter le voile et commencer une autre civilisation. S'il n'y a pas de socle et si un pays ne veut plus être lui-même, comment peut-on intégrer les étrangers ? Corneille a tout dit : "Si vous n'êtes pas Romain, soyez digne de l'être ». Si l'on n'est pas Français, il faut être digne de l'être. C'est cela l'intégration. [...] (Authier 2004).

15. Par ailleurs, ce comportement discursif de Makine est remarqué par les journalistes dont l'un intitule son entretien avec l'auteur exilé « Politiquement incorrect » (propos recueillis par Arnould de Liedekerke, Le Figaro Magazine, février 2000).

16. «C'est seulement une sorte d'abri de chasse [...], pas question d'y amener qui que ce soit. [...] Vous tenez vraiment à me rencontrer là où je passe mes vacances? Eh bien, suivez-moi » (Roignant 1996 ; les questions sont ici omises).

17. Nous n'avons aucune preuve de l'existence de cette autorisation; nous supposons cependant que la publication est autorisée par l'auteur s'il ne la réfute et ne la dément pas.

18. L'ethos du nomade est aussi repérable dans les romans d'Andreï Makine qui a créé plus d'un personnage sans domicile fixe, passant d'un lieu à l'autre, changeant d'adresse et de pays.

19. Dans le roman, ce même ethos d'exilé nomade est au contraire embarrassant car il empêche le protagoniste fictionnel de se rapprocher des autres, de tisser des liens et mène à une faiblesse sentimentale. 


\section{RÉSUMÉS}

Le présent article se penche sur les entretiens d'auteurs exilés, pour interroger la spécificité de l'image d'auteur qui s'y met en place. Il s'agit d'articuler l'entretien d'auteur et la notion de paratopie, c'est-à-dire le rapport simultané et paradoxal d'appartenance et de non-appartenance qu'entretient l'auteur avec la société d'accueil et le champ littéraire au sein duquel il écrit. Cette articulation s'effectue à partir des cas emblématiques de deux écrivains exilés en France, en l'occurrence Andreï Makine et Nancy Huston, qui écrivent leurs œuvres en français et participent ainsi à la vie littéraire française. Nous montrons que, par le biais de l'embrayage paratopique, les deux écrivains construisent une image de soi à multiples visages, tous unis sous le signe de la paratopie : l'ethos de «diseur de vérité » ou de «vagabond» de Makine, l'ethos d'une adepte du multiculturalisme ou d'une "exilée déracinée » de Huston, enfin, un ethos critique qui les place dans une paratopie de différents ordres - identitaire, spatiale, créatrice. C'est ce non-lieu qui leur permet de convertir leur intenable situation d'exilé en un lieu où la prise de parole et de position devient possible au point que le fait qu'ils soient étrangers n'est plus considéré comme un défaut ou un manque. Il s'agit alors d'un statut avantageux qui élargit leur champ de vision et leur potentiel créateur.

This article examines the exiled authors' interviews, in order to analyze the specificity of the authors' image that is worked out during the interview. Its objective is to demonstrate the connection between the authors' interviews and the concept of "paratopy" defined as the impossible belonging of the exiled authors to the French society and the literary field in which they write in a foreign tongue. This analysis is based on two emblematic cases of writers who have immigrated to France, namely Andreï Makine and Nancy Huston, who write their works in French and thus contribute to the French literary field. We show that both writers construct a multi-faceted self-image, all united under the banner of "paratopy": Makine's ethos of "truth teller" and "vagabond", Huston's preference for multiculturalism and an "uprooted exile", that places them in different kinds of "paratopies" - identitary, spatial, and creative . It is this nonplace which allows them to turn their unbearable situation of exile into a situation where the fact that they are foreigners is not considered a defect or a disadvantage, but quite on the contrary, widens their field of vision and creative potential.

\section{INDEX}

Keywords : ethos, exile, identity, literary field migration, paratopy, writer's in exile

Mots-clés : champ littéraire écrivain exilé, ethos, exil, identité, migration, paratopie

\section{AUTEUR}

\section{VALERIA PERY-BORISSOV}

Université Bar-Ilan, ADARR 